\title{
Research progress of chiral ligand exchange stationary phases in the enantiomer resolution
}

\author{
Hui Bai ${ }^{*}$ \\ ${ }^{1}$ School of Pharmaceutical Science and Technology, Tianjin University, 92 Weijin Road, Tianjin, 300072, China.
}

\begin{abstract}
The structure of chiral drugs contains at least one asymmetric center. When the enantiomers act on the human body, they are recognized by chiral receptors and enzymes in vivo, which will show different physiological effects and even adverse reactions. Therefore, it is very important for the development of chiral pharmacy to obtain chiral enantiomers with a single configuration by racemic resolution. Some general impurities will be introduced in the production of chiral drugs, thus the detection of impurities is also a crucial step in the quality control of chiral medicines. The chiral ligand exchange stationary phase has a strong recognition effect on enantiomer analytes with multiple chelating sites, and is very suitable for the separation and control of biological samples such as amino acids. In this work, the development of chiral ligand exchange stationary phases in enantiomeric resolution is reviewed, which is expected to provide a basis for the quality control of complex chiral drug components.
\end{abstract}

\section{Intoduction}

Chirality widely exists in nature and represents an essential feature of symmetry. If a compound is different from its mirror-image, it is called "chiral", while these two compounds are called a pair of enantiomers. The term chirality is more commonly used in the field of chemistry and medicine. When a chiral center is introduced into the molecular structure of medicine, the resulting pair of mirror-image enantiomers are called chiral drugs. More than $50 \%$ of the existing synthetic pharmaceuticals have chiral asymmetric centers. A pair of enantiomers is like human hands, they look very similar, but not the same. When a chiral drug enters the body, it is recognized by various chiral receptors, enzymes and carriers. Because the two enantiomers of chiral drugs have different stereostructure, the action of enantiomer in vivo will produce different pharmacokinetics and pharmacodynamics. ${ }^{[1]}$

The different pharmacological activities and even toxic side effects of chiral enantiomers can be reflected in the following areas: (1) Drug has the same enantiomeric activity but differs in the degrees of efficacy. For example, (S)-ketamine has $1 / 3$ of the analgesic effect of (R)ketamine, but it has a stronger hallucinogenic effect. (2) One type of enantiomer has a remarkable pharmacological effect, while the other type of enantiomer has low or no pharmacological activity. For example, L-lysine is physiologically active, while D-lysine can not be absorbed by the body. ${ }^{[2]}$ (3) The two enantiomers have different activities and acted as diverse drugs for treating various diseases. (2s, 3R)-propoxyphene (dextropropoxyphene) is a painkiller, and (2R, 3s)-propoxyphene (levopropoxyphene) is administered as a cough suppressant. ${ }^{[3]}$ (4) One of the enantiomers has a therapeutic effect, while the other has side effects or toxicity. For example, one enantiomer of ethambutol has pharmacological activity against tuberculosis, while the other has blinding side effects. ${ }^{[4]}$

Chiral pharmaceutical is to reasonably use the above differences in pharmacology and efficacy of chiral drugs to develop drugs with high effectiveness and few side effects. In clinical treatment, taking optically pure enantiomer can eliminate the toxic side effects caused by ineffective (harmful) enantiomers. This will be advantageous for researchers to control the pharmacokinetics and dosage better and improve the drug specificity. In 1992 and 1994, the United States and the European Union respectively promulgated the guidelines for chiral drugs, in which the Food and Drug Administration (FDA) required pharmaceutical companies to quantify the pharmacodynamic and toxicological effects of each enantiomer in future research and development of new drugs. ${ }^{[5]}$ With the development of new drugs, there are more than 600 chiral drugs among the more than 2000 synthetic drugs, less than 100 are active single-enantiomer drugs, and more than 500 racemic drugs for the rest. ${ }^{[6]}$ In view of the different pharmacological activities of chiral enantiomers, more attention has been paid to the development of singleenantiomers with high optical purity. ${ }^{[7]}$ In recent years, the number of racemic drugs approved for marketing has decreased. Therefore, obtaining optically pure enantiomers by means of control in drug research and development can promote the progress of the chiral pharmaceutical industry. In addition to controlling the direct synthesis of enantiomers in a single configuration in the research and development of new drugs, it is also an

\footnotetext{
*Corresponding author: bai_hui@tju.edu.cn.
} 
important issue for researchers to select suitable methods for the effective resolution and quality control of chiral drugs to promote the development of chiral pharmaceuticals.

At present, there are several methods to obtain chiral compounds with single enantiomers, such as natural sources, chemical synthesis and racemate resolution. More than 7,000 compounds have been isolated since Louis Pasteur reported the first optical enantioseparation in 1848. The racemate resolution has been widely used because of its low cost and simple process route, avoids using existing chiral molecules and omits the process of selecting suitable catalysts. The methods of racemate resolution mainly include crystallization resolution, chemical resolution, enzyme resolution and chromatographic resolution.

In the chromatographic separation method, a chiral environment (asymmetric center) can be introduced to make the enantiomers of drugs different in physical and chemical properties, so as to achieve separation. Compared with the classical enantiomer resolution method, chromatography has many apparent advantages and can meet enantiomer separation and determination requirements under various conditions. It can carry out rapid qualitative and quantitative analysis and achieve separation and micro-determination on the preparation scale. ${ }^{[8]}$

High-performance liquid chromatography (HPLC) will not change the solute configuration and lose its biological activity due to high temperature, and has high column capacity, which has excellent potential to develop into experimental and industrial-scale enantiomer preparation and separation. ${ }^{[9]}$ The separation methods of drug enantiomers by HPLC include the chiral derivatization reagent method, chiral mobile phase method and chiral stationary phase method. Chiral stationary phases (CSP) in the HPLC have developed rapidly in recent years due to the direct interaction with solute, simple separation methods, and high reliability of the quantitative analysis. ${ }^{[10]}$ The commonly used CSPs mainly include polysaccharide, Pirkle-type, crown ether, and ligand exchange CSPs. However, it is necessary to select the appropriate chiral column according to the sample structure. The chiral ligand exchange stationary phases present better binding ability and selectivity of compounds with chelating sites, which are suitable for the resolution of amino acids and other bio-related samples.

Because of the superior medical effect and increasing market share of chiral drugs, it is essential for the development of chiral pharmaceuticals to separate racemates by sensitive and straightforward means. In this paper, the research progress of ligand exchange chromatography stationary phase in chiral drug separation was reviewed, so as to provide support for designing a reasonable stationary phase structure to realize the separation of complex chiral drug components. Meanwhile, it can also promote the development of the chemical industry, pharmaceutical industry and biological industry related to chirality.

\section{Chiral ligand exchange stationary phases}

\subsection{Chiral ligand exchange chromatography (CLEC)}

Chiral ligand-exchange chromatography (CLEC), which introduces a specific metal ion and a particular chiral ligand into the chromatographic system, can form a pair of diastereomeric ternary complexes with chiral enantiomers. Then the stereoselective separation of enantiomers can be realized by the chromatographic process. In the middle of the last century, metal-organic coordination chemistry was widely used in the field of chemical synthesis. In 1961, Helfferich first proposed the concept of "ligand exchange". After that, through the further development of Rogozhin and Davankov, the complete separation of chiral enantiomers by liquid chromatography was successfully realized, which also proved the practical application value of ligand exchange chromatography, and thus defined ligand exchange chromatography accurately. ${ }^{[11]}$

CLEC should be defined as a process. In this process, the interaction between the stationary phase and the molecules to be separated occurs when the central ions form a coordination bond in the complex range. Therefore, unlike adsorption chromatography and other types of chromatography, CLEC does not directly act on the stationary phase, it is realized by the exchange of central metal ions with their suitable ligands, so it is called chiral ligand exchange chromatography.

\subsection{Chiral recognition mechanism in CLEC}

The process of chiral resolution is a recognition between the chiral stationary phase and chiral enantiomer. In 1952, Dalgliesh $^{[12]}$ proposed a "three-point interaction" chiral recognition model for direct chromatographic separation of amino acid enantiomers using paper chromatography. "Three-point interaction" is the original description of the asymmetric recognition condition. In the chiral recognition, as shown in Fig.1, there are three possible interaction sites between chiral enantiomers and chiral selectors on the stationary phase. All three sites can be combined when the enantiomer I interact with the CSP, while the enantiomer II can only form the interaction of A-A' and B-B'. If the interaction of C-C' leads to the stability of the transient diastereomeric complex between the solute and the CSP, the enantiomer I will form an ideal fit. The stability constant of enantiomer I is higher than that of enantiomer II, a pair of enantiomers can get a chiral resolution as a result.
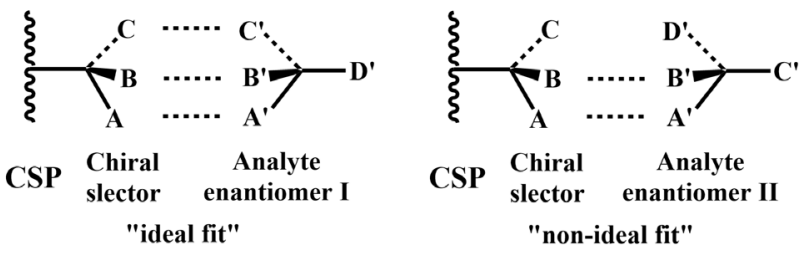

Fig 1. "Three-point interaction" model of chiral recognition 
At present, the "three-point interaction" model has been successfully applied to chiral liquid chromatography, and a series of CSPs based on different interactions have been developed and successfully applied to the analysis of various chiral drugs. Unlike other CSPs, The chiral recognition on chiral ligand exchange stationary phase is a process of metal complexation equilibrium. As shown in Fig.2, chiral selectors and analytes are adsorbed in the coordination spheres of the central metal ions to form a pair of diastereomeric metal complexes with different stability, thus obtaining chiral resolution. Because of the special recognition mechanism, the chiral ligand exchange CSPs are especially suitable for the analysis of enantiomers with multiple chelating sites, such as amino acids and hydroxy acids.

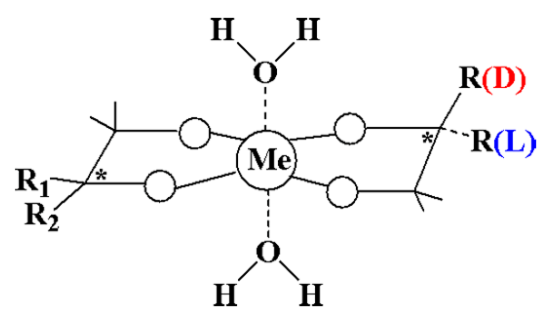

Fig 2. Coordinate bond of the chiral selector, metal ion, and enantiomers in CLEC

CLEC combines the characteristics of ion exchange and coordination chemistry, so it can realize the work which any of the above processes alone can not accomplish. Since Davankov and Rogozhin successfully achieved the separation of amino acid enantiomers by CLEC in the late 1960s, the ligand exchange stationary phase has developed rapidly in recent years. CLEC has become a new chiral liquid chromatography technology that can completely and stably separate chiral enantiomers. A series of native and functionalized ligand exchange CSPs with different matrices have been synthesized.

\subsection{Development of chiral ligand exchange stationary phases}

The ligand exchange chiral stationary phases usually select chiral amino acids or their derivatives as the chiral selectors to bond to the solid carrier (such as silica gel) and form metal ion complexes with divalent metal ions. The metal ions can be $\mathrm{Cu}$ (II), $\mathrm{Zn}$ (II), and Ni (II), among which $\mathrm{Cu}$ (II) has the highest selectivity and is the most versatile. The enantiomers and stationary phase can form two non-enantioselective ternary complexes, which differ in the thermodynamic and kinetic stability to express different chromatographic behaviors, thus the chiral resolution can be realized.

Davankov et al. ${ }^{[13]}$ bonded heterocyclic L- $\alpha$-amino acids (proline and azetidine carboxylic acid) on the styrene-divinylbenzene resin to achieve chiral separation of racemic amino acids, as shown in Fig.3. The results showed that the sorbent containing proline had higher enantioselectivity than the one with azetidine carboxylic acid, which indicated that the existence of a fivemembered heterocyclic ring could improve the chiral selectivity of stationary phases. However, the CSPs based on styrene-divinylbenzene resin performed a relatively lower column efficiency.
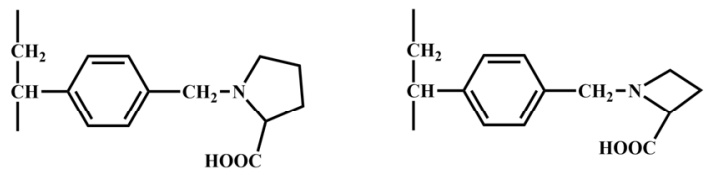

Chiral selector---L-proline Chiral selector---L-azetidine carboxylic acid

Fig 3. Chemical structures of chiral ligand stationary phases with different chiral selectors

Toyohide et al. ${ }^{[14]}$ bonded L-hydroxyproline with porous silica gel and got a better column efficiency on the separation of chiral amino acids. They also used chiral stationary phases in series with various hydrophobic columns to obtain good results in the separation of racemic dansyl amino acids. They also used chiral stationary phases in series with different hydrophobic columns and received good results in separating racemic dansyl amino acids. However, many attempts on chiral and hydrophobic column types are needed to get satisfactory results, thus the enantiomeric resolution can not be easily achieved.

Other scholars have also explored the improvement of the selectivity of chiral ligand exchange chromatography stationary phases, and have made some progress. Zhu et al. ${ }^{[15]}$ synthesized L-proline stationary phases with different surface coverage. The resolution results of $\alpha$ amino acids show that the stationary phases with different chiral ligand densities have significant differences in chiral resolution. However, due to the poor selectivity and single-mode retention of these CSPs, it is difficult to separate a variety of amino acid racemates with different hydrophobic simultaneously.

Unlike the single-mode recognition, mixed-mode chromatography (MMC) is a separation technique that contains two or more retention mechanisms. ${ }^{[16]}$ Because of the multiple mechanisms of interaction on solute retention, MMC can significantly improve the separation selectivity of the stationary phases, especially for the analysis of complex samples. Due to the limitation of chiral resolution in chiral ligand exchange stationary phases with single chiral selectors or single functional mechanism, MMC has attracted people's attention for its high selectivity and suitability for the separation of complex solutes. Additional forces such as hydrogen bonding, dipoledipole interaction and hydrophobic interaction can be introduced to the separation mechanism of MMC. These secondary forces contribute to the chiral analysis and constitute the three-point interaction mechanism in chiral resolution.

Roccaldo Sardella et al. ${ }^{[17]}$ dynamically coated (S)trityl-L-cysteine (L-STC) on octadecyl silica to determine the D-amino acid content of cheese samples, improving the chiral selectivity through the introduction of the hydrophobic interaction. Fig.4. shows the proposed structure of the diastereomeric ternary complex formed between the CSP and L-Ala. In addition, the introduction of the $\mathrm{C}_{18}$ matrix significantly improved the sensitivity of the stationary phase to the detection of general impurities. Therefore, it is more feasible to design and construct a mixed-mode chiral stationary phase with stronger 
cooperative effects to achieve the separation of impurities and chiral enantiomers at the same time. This can promote the research of the chiral ligand exchange stationary phase in the quality control of chiral drugs.

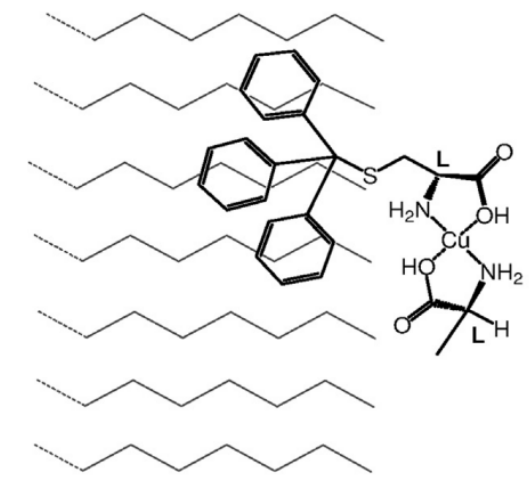

Fig 4. The proposed structure of the diastereomeric ternary complex formed from the fixed ligand (L-STC), $\mathrm{Cu}(\mathrm{II})$ and L-Ala

\section{Conclusion and outlooks}

At present, the market share of chiral drugs is increasing day by day. Because of the particularity of chiral medicines, the difference in pharmacological effects of a pair of enantiomers will affect the clinical use of drugs. Therefore, the development of the chiral pharmaceutical industry has been widely concerned by scholars. We should pay attention to the resolution of chiral enantiomers and focus on the detection and separation of general impurities in chiral drugs.

In the methods of enantiomeric separation, HPLC can meet the requirements of determination and chiral resolution under various conditions, and plays a crucial role in the quality control of chiral drugs. The chiral stationary phases have the advantages of wide application, simple preparation and high reliability of the quantitative analysis. In recent years, the diversity of chiral stationary phases has made it suitable for analyzing various solutes. The chiral ligand exchange stationary phase has been widely used to separate amino acids, amino alcohols, and other biologically related compounds due to its specific selectivity. According to the researches of scholars in recent years, the ligand types that can improve the chiral selectivity have been screened out. In addition, current research shows that the introduction of secondary forces such as hydrophobic interaction can affect the binding sites and modes of the CSP and the analyte. The influence of other interaction modes (such as hydrogen bond, dipole-dipole interaction) on chiral recognition is also worth study in the future. It is expected that the design of the chiral stationary phases with multiple forces by this mean will improve the sensitivity of chiral columns to non-chiral solutes and be beneficial for the analysis of general impurities in chiral pharmaceuticals. Therefore, it is speculated that the investigation of chiral ligand exchange stationary phase with multi-action mode can broaden its application market, which is conducive to the development of economic and reliable schemes for chiral drug quality control.

\section{References}

1. T.P. Yoon, E.N. Jacobsen, Sci., 299, 1691-1693, (2003)

2. H. Zahradnickova, P. Husek, et al., Anal. Bioanal. Chem., 388, 1815-1822, (2007)

3. O. Wahl, U. Holzgrabe, Talanta, 27, 249-255, (1994)

4. Q.Q, Huanf, R. He, et al. Curr. Trends. Med. Chem., 11, 810-818, (2011)

5. H.G. Su, Q.M. Zhou, Y.Q. Shen, Chinese Journal of Experimental Traditional Medical Formulae, 24, 201208, (2018)

6. H.Y. Aboul-Enein, N. Bounoua, Chirality, 33, 196208, (2021)

7. H.Y. Aboul-Enein, I. Ali, Chromatographia, 54, 200202, (2001)

8. A.R. Ribeiro, A.S. Maia, et al., J. Chromatogr. B Analyt. Technol. Biomed. Life Sci., 968, 8-21, (2014)

9. G. Junichi, G. Nobuharu, et al., J. Chromatogr., 239, 559-564, (1982)

10. A. Tarafder, L. Miller, J. Chromatogr. A, 1638, 461878, (2021)

11. V.A. Davankov, S.V. Rogozhin, J. Chromatogr. A, 60, 280-283, (1971)

12. I.W. Wainer, M.C. Alembik, J. chromatogr., 367, 59$68,(1986)$

13. V.A. Davankov, Y.A. Zolotarev, J. Chromatogr. A, 155, 295-302, (1978)

14. T. Toyohide, A. Homare, I. Daido, J. Chromatogr. A, 407, 151-158, (1987)

15. X.Y. Zhu, X.Q Han, Se Pu, 2, 223-226, (2002)

16. I. Tanret, D. Mangelings, J. Chromatogr. Sci., 47, 407-417, (2009)

17. S. Roccaldo, L. Antonella, Food Control, 34, 478-487, (2013) 